\title{
Politikfragen und nachhaltige Entwicklung als zentrale Kohärenzprobleme
}

Synthesebericht

Monika Egger and Jacques Forster

\section{OpenEdition}

\section{Journals}

Electronic version

URL: http://journals.openedition.org/sjep/1318

DOI: $10.4000 /$ sjep.1318

ISSN: 1663-9677

\section{Publisher}

Institut de hautes études internationales et du développement

Printed version

Date of publication: 1 février 1995

Number of pages: $206-220$

ISSN: 1660-5926

\section{Electronic reference}

Monika Egger und Jacques Forster, «Politikfragen und nachhaltige Entwicklung als zentrale Kohärenzprobleme », Schweizerisches Jahrbuch für Entwicklungspolitik [Online], 14| 1995, Online erschienen am: 05 Mai 2013, abgerufen am 08 September 2020. URL : http://journals.openedition.org/ sjep/1318 ; DOI : https://doi.org/10.4000/sjep.1318 


\title{
Politikfragen und nachhaltige Entwicklung als zentrale Kohärenzprobleme
}

\author{
Synthesebericht
}

\author{
Monika Egger und Jacques Forster
}

Kohärenz: Zusammenhang; die gleiche Wellenlänge und Schwingungsart haben (Physik)

Kohärenzprinzip: Grundsatz von dem Zusammenhang alles Seienden (Philosophie)

(Der Grosse Duden)

Die Frage nach der Kohärenz bzw. der Inkohärenz in den bilateralen Beziehungen der Schweiz mit Indonesien macht deutlich, wie vielschichtig die Problematik gelagert ist und wie zahlreich die Aktions- und Interventionsebenen sind. Die nachfolgende schematische Darstellung der Problembereiche, wie sie in den verschiedenen Beiträgen genannt wurden, verdeutlicht dies (siehe Abbildung „Kohärenzprobleme“). Die genannten Kohärenzprobleme in der Schweiz und in Indonesien sind nach den aussenpolitischen Zielen eingeordnet.

Das Schwierigste in der Kohärenzfrage ist - wie immer bei komplexen Fragenstellungen - die Prioritätensetzung. Die Wahl des zentralen Kohärenzproblems bot den Einstieg in die Kohärenzdiskussion am runden Tisch: „Welches ist für mich das wichtigste Kohärenzproblem in den Beziehungen zwischen der Schweiz und Indonesien? Welches sind für mich die Dialogpartner, die Akteure, um das in meinen Augen wichtigste Kohärenzproblem zu lösen ?" Dabei soll als Resultat vor Augen gehalten werden, welche Erwartungen ich an erhöhte Kohärenz in einem Schlüsselbereich stelle und welchen konkreten Beitrag ich für die Optimierung des Handelns leiste.

Die Mehrheit der Teilnehmerinnen und Teilnehmer des Seminars nannten zwei prioritäre Themenblöcke, einen politischen Bereich und einen wirtschaftlichen:

1. Demokratie, Demokratisierungsprozess, „Good Governance“, die Menschenrechtslage in Indonesien;

2. die Ausgestaltung eines nachhaltigen Wirtschaftswachstums im Land. 
Für eine bessere Kohärenz müssen alle in der Darstellung genannten Akteure handeln, weil die Aktionsebenen ineinandergreifen. Mehr als bisher müssen nebst der Zentralregierung andere Partner verstärkt gesucht werden.

„Wir haben bis jetzt primär mit der Zentralregierung gearbeitet. Es scheint mir wichtig, dass wir jetzt auch andere Partner suchen, dass die Privatwirtschaft vermehrt als Partner auftritt, und zwar nicht nur für den Export aus der Schweiz, sondern auch im Bereich Privatinvestitionen, dass es die Schweizer

\section{Abbildung: Kohärenzprobleme im Rahmen der aussenpolitischen Ziele der Schweiz und Akteure}

\begin{tabular}{|c|c|c|}
\hline & Indonesien & Schweiz \\
\hline $\begin{array}{l}\text { Sicherheit + Frieden, } \\
\text { Rechtsstaat/Demokratie } \\
\text { Menschenrechte }\end{array}$ & $\begin{array}{l}\text { Korruption } \\
\text { Autoritärer Staat } \\
\text { Menschenrechte } \\
\text { Ethnisch-religiöse Konflkte } \\
\text { Good governance } \\
\text { Rüstungskontrolle }\end{array}$ & $\begin{array}{l}\text { Waffenexporte } \\
\text { Rechtshilfe } \\
\text { Good governance } \\
\text { Rüstungskontrolle }\end{array}$ \\
\hline Wohlfahrt & $\begin{array}{l}\text { Dualistische Wirtschaft } \\
\text { Arbeitsmarkt } \\
\text { Gewerkschaftsrechte }\end{array}$ & $\begin{array}{l}\text { Handel und } \\
\text { Direktinvestitionen } \\
\text { Sozialklauseln } \\
\text { Entwicklungs- } \\
\text { zusammenarbeit } \\
\text { Exportrisikogarantie } \\
\text { Fluchtkapital } \\
\text { Patentrecht } \\
\text { Doppelbesteuerungs- } \\
\text { abkommen }\end{array}$ \\
\hline $\begin{array}{l}\text { Abbau sozialer } \\
\text { Gegensätze }\end{array}$ & $\begin{array}{l}\text { Gender balanced } \\
\text { promotion } \\
\text { Armutsstrategie }\end{array}$ & $\begin{array}{l}\text { Entwicklungs- } \\
\text { zusammenarbeit } \\
\text { Armutsstrategie }\end{array}$ \\
\hline Umwelt & $\begin{array}{l}\text { Troppenholzraubbau } \\
\text { Energiepolitik } \\
\text { Umweltzerstörung }\end{array}$ & $\begin{array}{l}\text { Energiepolitik } \\
\text { Tropenholzboykott } \\
\text { Umweltzerstörung }\end{array}$ \\
\hline \multicolumn{3}{|c|}{ Akteure } \\
\hline \multicolumn{3}{|c|}{$\begin{array}{l}\text { Regierung/Nerwaltung/Armee } \\
\text { Privatwirtschaft/Gewerkschaften } \\
\text { Hilfswerke/NGO/Kirchen } \\
\text { Wissenschaft } \\
\text { e Partner (UNO, EU, IWF/Weltbank, Asean) } \\
\text { Andere bilaterale Partner }\end{array}$} \\
\hline
\end{tabular}


Wirtschaft vermehrt interessiert, auch in Indonesien Arbeitsplätze zu schaffen“ (Robert Jenny). „Die Situation der Frauen muss gestärkt werden. Ich verspreche mir am meisten bei der "Gender balance promotion" durch die NGO. Ich glaube, die staatlichen Organe sind noch sehr, sehr weit weg von der Realität“ (Margrit Meier).

Die Problemgewichtung sowie mögliche Lösungsansätze fielen je nach Standpunkt differenziert aus: Auffallend übereinstimmend - um nicht zu sagen harmonisch - fiel die Beurteilung der politischen Fragen (Menschenrechtslage) in Indonesien und die Notwendigkeit zu mehr Demokratie und für eine bessere Regierungsführung aus. Widersprüchlicher und emotionsgeladener waren die Voten über die Kohärenz in den Wirtschaftsbeziehungen zwischen der Schweiz und Indonesien mit dem Ziel der nachhaltigen Entwicklung. Wobei unter nachhaltiger Entwicklung das Gewicht stark auf das sozial ausgewogene Wirtschaftswachstum gelegt wurde. Weniger beleuchtet wurde die Umweltproblematik.

\section{Bessere Kohärenz durch bessere Politik - Demokratisierung, Good Governance, Menschenrechte}

\section{Bilaterale Beziehungen}

Die Diskussion der politischen Dimension der Kohärenzfrage stand unter dem Eindruck des in den vorangegangenen Tagen durch die USA gefällten Entscheides, in den Beziehungen mit China die Menschenrechtsfrage in Zukunft strikte zu trennen von der Handelspolitik. So stellte sich einleitend auch für die Seminarteilnehmer die Frage, ob die Schweiz ihre Wirtschaftsbeziehungen mit einem Land abbrechen soll, wenn dieses die Menschenrechte nicht respektiert oder. Ist Indonesien ein solches Land?

„Wir haben im Spannungsfeld von Menschenrechten und Aussenwirtschaftspolitik nicht besonders raffinierte Mechanismen. Lange werden Probleme totgeschwiegen, und wenn ein Problem der Inkohärenz auftritt, gibt es praktisch nur zwei Reaktionsmöglichkeiten: Es wird einerseits der sofortige Abbruch der Wirtschaftsbeziehungen gefordert, wenn sich beispielsweise ein schwerwiegendes Menschenrechtsproblem stellt, und die Wirtschaft erklärt dann ihrerseits, warum man das nicht machen soll“ (Peter Maurer).

Um aus der Situation herauszukommen, dass sich in der Frage der Beziehungen mit politisch fragwürdigen Regierungen zwei entgegengesetzte Standpunkte in der Öffentlichkeit festfahren, wird übereinstimmend mehr Dialog gefordert, und zwar ein regelmässiger, vernetzter Dialog zwischen NGOs, Industrie und Verwaltung in der Schweiz darüber, wie eine kohärente Aussenwirtschaftspolitik und Entwicklungspolitik im Falle von Menschenrechtsverletzungen aussehen muss. Eine grundsätzliche Haltung müsste herausgearbeitet werden, die jeweils an die spezifische Situation eines Landes angepasst werden könnte. Dies bedeutet, Differenzierungen vorzunehmen je nach Situation 
und Land, jedoch ohne den Fehler zu machen, zwischen verschiedenen Ländern verschiedene Standards anzuwenden, je nach Eigeninteresse der Schweiz und je nach Gewicht der Aussenwirtschaftsbeziehungen und der gewährten Entwicklungshilfe. "Ich denke, man muss aber aufpassen, dass man nicht zwischen verschiedenen Ländern verschiedene Standards appliziert, nur weil man an einem Ort mehr Leverage hat als am anderen Ort" (Hans-Peter Maag).

Es herrscht mehrheitlich die Einsicht beim Rundtischgespräch, dass die kleine Schweiz politisch einen geringen Einfluss auf ein Land wie Indonesien hat. "Indonesien ist zwar ein Schwerpunktland in Sachen Entwicklungszusammenarbeit für die Schweiz, die Schweiz ist aber bei weitem kein Schwerpunktpartner der Indonesier, weder in der Entwicklungszusammenarbeit, noch auf der politischen Ebene. Kommt hinzu, dass Indonesien zu der Kategorie Länder gehört, die es sich leisten können, sich von Konditionalitäten oder irgendwelchem internationalem Druck in Sachen Menschenrechten, Good Governance, Demokratie nicht beeindrucken lassen zu müssen." (Konrad Specker).

Trotz der Relativierung dessen, was die Schweiz bewirken kann, herrscht Konsens darüber, dass eine bessere Aussenwirtschaftspolitik der Schweiz im Zusammenhang mit Menschenrechtsverletzungen angestrebt werden soll. Die Aussicht auf einen geringen Impakt soll uns nicht zur Resignation und zum Nichtstun verleiten. Aktivitäten von schweizerischen Exporteuren sind zu verhindern, wenn sie Menschenrechtsverletzungen Vorschub leisten (Waffenexporte). Generell muss vermehrt darauf geachtet werden, dass die schweizerischen Wirtschaftsbeziehungen nicht Kräften dienen, die korrupt sind, und letztlich die Demokratie behindern. Es gibt eine einfache Möglichkeit, die Bedürfnisse in einem Partnerland abzuklären, indem wir die durch unsere Wirtschaftsbeziehungen und durch die Entwicklungshilfe betroffenen Menschen fragen, sie um inre Interpretation unserer Vorstellungen von Wirtschaftsbeziehungen, Good Governance und Menschenrechten bitten: „Was erwartet Ihr von der Schweiz ? Von den schweizerischen Hilfswerken? Vom Staat? Von der Privatwirtschaft ? Wir müssen keine Angst vor diesen Fragen haben, weil die Antworten nach meinen Erfahrungen in der Regel aus ihrer persönlichen Betroffenheit heraus viel differenzierter ausfallen, als einfach der Ruf nach einem Boykott beispielsweise" (Richard Gerster).

Durch besseren Dialog mit privaten und öffentlichen Partnern in Indonesien auf allen verschiedenen Ebenen - Regierung, Verwaltung in der Zentrale aber vermehrt auch in den Regionen und Distrikten - leisten wir auch der Projektion unserer Demokratie- und Kulturbegriffe auf andere Staaten Vorschub. Bessere direkte Information aus den verschiedenen Gesellschaftsschichten hilft uns, unsere Sicht über die politische Lage zu klären und unsere Möglichkeiten für Verbesserungen einzubringen. Auch unsere Vorstellung über die ideale wirtschaftliche Entwicklung müssen differenziert und länderweise angepasst werden. Kontrovers diskutiert wurde die These, wie sie Jean-Luc Maurer in seinem Beitrag vertritt, dass es zuerst den autoritären Staat braucht, um auf ein gewis- 
ses Entwicklungsniveau zu kommen, von wo aus sich dann demokratische Kräfte von selbst entwickeln. Dieser Sicht wird widersprochen. Wir sollten historische Rückschau halten über die Entwicklung in Europa, wo sich die Gesellschaften demokratisch und wirtschaftlich entwickelt haben. „Der Zusammenhang zwischen wirtschaftlicher Entwicklung, Wohlstand und Demokratie ist ein Wechselwirkungsverhältnis. Sowohl Demokratie als auch wirtschaftlicher Wohlstand schaukeln sich beide gegenseitig auf in komplexen Zusammenhängen“ (Günther Bächler).

Menschenrechtsverletzungen dürfen nicht toleriert werden durch Schweigen; sie müssen auf diplomatischer Ebene thematisiert werden. Auch die Privatwirtschaft sollte sich Möglichkeiten ausdenken, wie sie, nicht als einzelne Firma, aber über einen Verband beispielsweise ihrem Missfallen über konkrete Fälle von Menschenrechtsverletzungen Ausdruck gibt und den Partnern mitteilt, dass dies längerfristig gegen die Grundsätze in den Wirtschaftsbeziehungen verstösst, wie sie für richtig angesehen werden. Indonesien sei schliesslich darauf angewiesen, langfristig als politisch stabil zu gelten, wenn es vermehrt Auslandinvestitionen anziehen will. Eine Bedingung für langfristige politische Stabilität ist auch die Respektierung der Menschenrechte im Land. Dieser Ansicht widerspricht die in der Privatwirtschaft weitverbreitete Meinung, dass ein repressives System nicht gleich auch ein schlechtes Investitionsklima für private Investoren bedeutet, zumindest kurzfristig nicht. Wenn Investoren vor der Wahl stehen, ziehen sie wahrscheinlich repressive Systeme unsicheren, bürokratisch aufgeblähten, demokratischen Systemen vor. Dies gilt es zu bedenken, wenn die Rolle der Privatwirtschaft im Ausbau der Kohärenz diskutiert wird. Es braucht noch viel Überzeugungsarbeit in diese Richtung. Denn für die Privatwirtschaft können noch mehr Militärausgaben gleichbedeutend sein mit Erhaltung von - repressiver - „Ruhe und Ordnung“ und somit die Investitionssicherheit aufrechterhalten.

Menschenrechtspolitik soll mehr sein als ein deklamatorisches Anhängsel von Wirtschaftszusammenarbeit; sie muss auch jenseits der symbolischen Politik konkret greifen. Positive Massnahmen sind nicht zu reduzieren auf einen „spill over effect“ der Wirtschaft. Konkrete positive Massnahmen könnte beispielsweise die Ausarbeitung und Anwendung eines Verhaltenskodexes (Code of Conduct) der schweizerischen Industrie im Demokratisierungsprozess eines Landes sein, in dem der Beitrag der Privatwirtschaft für die Entwicklungszusammenarbeit definiert wird, die Gleichgerichtetheit der privaten mit den öffentlichen bilateralen Beziehungen, usw. Allgemein wird „etwas weniger Leisetreterei der Schweiz" gewünscht und ein "spürbarer Gesinnungswandel in der Politik" (Robert Jenny).

Allerdings dürfen wir unsere eigene Entwicklungsgeschichte und die damit verbundenen Ungerechtigkeiten nicht verdrängen. Vorsicht ist auch geboten in der Sprache. „Ist das 'autoritäre Regime' in Indonesien vergleichbar mit Chile unter Pinochet oder mit Spanien unter Franco ? Korruption, ja, aber nicht ausser Acht lassen, dass wir auch Korruption in der Schweiz und anderswo haben“ (Philippe Régnier). 
In der Kohärenzdiskussion wird die Glaubwürdigkeit der Länder des Nordens im Politik- und im Wirtschaftsdialog mit den Ländern des Südens zur zentralen Bedeutung. Sind wir mit unserem hohen Ressourcenverbrauch glaubwürdig, wenn wir den Entwicklungsländern Mässigung im Energieverbrauch zur Auflage für eine nachhaltige Entwicklung machen ? Ist die Schweiz glaubwürdig, wenn sie sich in einem Fall UNO-Wirtschaftssanktionen anschliesst (Haiti) und im anderen Fall nicht (Südafrika) ? Auch die nationale Glaubwürdigkeit stellt sich in der Schweiz: „Sind unsere Bestrebungen nach Kohärenz überhaupt glaubhaft, wenn bei Volksabstimmungen alle aussenpolitischen Vorschläge der Regierung öffentlich abgelehnt werden ?" (Robert Jenny). Wie gehen wir um mit den grossen Divergenzen zwischen Regierung, Parlament und Volk in aussenpolitischen Fragen.

\section{Kohärenz im aussenpolitischen Umfeld}

Das Schwergewicht der Kohärenzdiskussion lag bis hierher auf der Ebene der bilateralen Beziehungen. Wie sieht eine kohärente schweizerische Zusammenarbeit mit Indonesien im internationalen Rahmen aus? Wie soll die Schweiz sich kohärent verhalten, wenn internationale Verhaltensregeln festgelegt werden, wie beispielsweise die Sanktionen der UNO gegenüber einem Land (erwähnte Beispiele Südafrika und Haiti) ?

Weltweit koordinierte oder parallel laufende Aktionen können durchaus eine Regierung in inrer Politik beeinflussen. Erwähnt wird eine internationale Kampagne, koordiniert von lokalen NGO in Malaysia vor einigen Jahren, welche erfolgreich den Gesetzgebungsprozess in Malaysia beeinflusst hatte. Die Regierung musste eine Gesetzesvorlage, welche die Rechte der privaten Organisationen zurückbinden wollte, fallenlassen.

Es bestehen Einrichtungen wie das Entwicklungshilfekomitee der OECD oder die Konsultativgruppen der Weltbank, in deren Rahmen die nationalen Politiken gegenüber Indonesien international abgestimmt werden. Gemeinsam formulierte Ziele und Strategien versprechen bessere Kohärenz.

Die wirtschaftliche Interdependenz, wie sie heute Realität ist, und wie sie in der nun angebrochenen Zeit der allgemeinen Liberalisierung weiter zunehmen wird, erlaubt es den einzelnen Staaten langfristig nicht mehr, je eigene, nationale Menschenrechtsstandards anzuwenden. „Die wirtschaftliche Interdependenz wird in zunehmendem Masse die Möglichkeiten des Nationalstaates unterlaufen, eigenwillig Menschenrechte anzuwenden. Wenn ein Land intensiven Handel treibt mit Ländern, die keine Gewerkschaftsrechte respektieren, dann wird es nicht mehr möglich sein, Gewerkschaftsrechte im eigenen Land aufrechtzuerhalten. Überspitzt formuliert könnte man sagen, selbst wenn es uns kulturell gesehen egal sein sollte, so kann es uns wirtschaftlich gesehen nicht egal sein, welchen Stellenwert die Arbeitnehmerrechte in Indonesien haben. Es lässt sich auf die Länge nicht aufrechterhalten, dass zwei komplett andere Systeme gelten" (Margrit Meier). 


\section{Bessere Kohärenz durch nachhaltiges Wirtschaftswachstum}

Welchen Beitrag leisten Entwicklungszusammenarbeit und Exportförderung für das nachhaltige Wirtschaftswachstum in Indonesien ? Oft - nicht immer stehen Entwicklungszusammenarbeit und Exportförderung in einem konfliktreichen Spannungsfeld zueinander. Dieser "traditionelle Konflikt“ in der Entwicklungszusammenarbeit dürfte in Zukunft abgelöst werden durch einen „policy mix“ (Instrumentenmix). Indonesien ist ein gutes Beispiel für ein Partnerland der schweizerischen Entwicklungszuammenarbeit - ein Schwerpunktland , wo die technische Zusammenarbeit abgelöst wird durch wirtschaftspolitische Massnahmen. Es besteht ein grosses Bedürfnis nach Modernisierung der Technologien und nach Privatinvestitionen. Indonesien muss seine Exporte ausbauen können, wenn das Land auch in Zukunft wirtschaftlich wachsen will.

„Die Frage der Investitionsförderung in Indonesien im Übergang von einer nicht arbeitsteiligen Wirtschaft zu einer sich wandelnden industriellen Gesellschaft ist ein Hauptthema, und es bietet eine sehr gute Möglichkeit, den traditionellen Gegensatz zwischen Entwicklungszusammenarbeit und Exportförderung zu überwinden, in dem Sinne nämlich, dass es Möglichkeiten gibt, diesen Investitionsprozess über öffentliche Mittel zu fördern, einen Investitionsprozess, der sowohl für schweizerische Firmen wie auch für indonesische Firmen von Vorteil sein kann und einen gesamtwirtschaftlichen Beitrag leistet" (Matthias Meyer). Ziel ist die Förderung eines effizienten Technologietransfers, welcher einen Beitrag zu nachhaltigem Wirtschaftswachstum leisten kann.

„Policy mix" bedeutet, verschiedene Instrumente der Entwicklungs- und Wirtschaftsbeziehungen parallel und sich gegenseitig ergänzend einzusetzen. In diesem Zusammenhang wird von einer Mehrheit der Tischrunde gefordert, dass die technischen Programme der DEH weitergeführt werden, denn Technologieförderung braucht ausgebildete Leute. Mehrheitlich wurde kein Verständnis aufgebracht für den Entscheid der DEH, sich aus Indonesien zurückzuziehen. Aufgrund der schweizerischen Prioritäten in der Entwicklungszusammenarbeit, die bei der Armutsbekämpfung und bei der Unterstützung der ärmsten Bevölkerungsschichten liegen, wird als wichtig erachtet, „den Industrialisierungsopfern etwas $\mathrm{zu}$ bieten und nicht in allgemein investitionsfördende Rahmenbedingungen zu investieren, wozu die Regierung selber die Möglichkeit hat" (Richard Gerster).

\section{Kohärentes Verhalten in Indonesien}

Als ebenfalls wichtige Frage der Kohärenz wurde gefordert, die indonesische Regierung auf ihre Rüstungsausgaben anzusprechen (erwähnt wurden Flugzeug- und andere Rüstungskäufe) und Alternativen für eine sinnvollere Verwendung der Mittel vorzuschlagen. Auch auf die fragwürdige Subventionspolitik müsste die indonesische Regierung hingewiesen werden, werden doch offenbar hohe Finanzmittel in marode Betriebe von Protegierten des Regimes gesteckt. Riesige Verluste werden mit Staatsmitteln abgedeckt. Bessere Kohä- 
renz könnte für die Schweizer Privatwirtschaft bedeuten, weniger Geschäfte mit dem Staat abzuwickeln und mehr in die privaten Betriebe zu investieren und mit innen zu kooperieren und den Transfer von angepasster Technologie im mittleren technologischen Feld und nicht im High-Tech-Bereich zu fördern. Der Instrumentenmix sieht tatsächlich die Unterstützung von Mittel- und Kleinunternehmen in Indonesien vor. Er will auch die Zusammenarbeit von entsprechenden Schweizer Betrieben mit indonesischen Betrieben fördern. Doch gelte es zuerst einmal, beim Abbau der hohen gegenseitigen Berührungsängste auf beiden Seiten zu vermitteln. Dieser Ansatz folgt der allgemeinen Erkenntnis, dass die Bedeutung der Rolle des privaten Sektors in der traditionellen Entwicklungszusammenarbeit bisher unterschätzt wurde. Das BAWI sieht seine Prioritäten in der Förderung von Umwelttechnologien und in der Förderung einer vom Privatsektor getragenen Ausbildung. Mischkredite werden in der Entwicklungszusammenarbeit mit Indonesien in der Zukunft wahrscheinlich keine grosse Rolle mehr spielen.

\section{Was bedeutet Kohärenz ?}

Die Diskussion über die beiden Bereiche politisch-soziale Fragen und wirtschaftliche Nachhaltigkeit ging immer von der Kohärenzfrage aus, und vielleicht verstanden die Teilnehmenden der Tischrunde nicht immer dasselbe unter Kohärenz. Deshalb wurde der dritte Teil der Diskussion auf die Fragen ausgerichtet: „Was verstehen Sie unter Kohärenz ?" und "Was erwarten Sie von diesem durch das Nord-Süd-Leitbild eingeleiteten Prozess des gemeinsamen Dialogs mit dem Ziel einer besseren Kohärenz ?"

Möglichst kurze, prägnante Antworten sollten in einer Tischrunde auch den Sinn der heutigen „Testrunde“ erhellen, d.h. den möglichen Nutzen eines Dialogs über gemeinsame Perspektiven unter verschiedenen Akteuren mit verschiedenen Interessen beleuchten und allenfalls den einzuschlagenden Prozess und das notwendige Instrumentarium aufzeigen.

Die meisten Teilnehmerinnen und Teilnehmer näherten sich der Definition der Kohärenz mit der relativierenden Feststellung, dass Widersprüche zum Leben gehören, ja das Leben ausmachen. Das gesellschaftliche und politische Leben ist widersprüchlich und unkohärent. Das Leben selbst ist voller Widersprüche. Ist es überhaupt sinnvoll, Widersprüche möglichst zu beseitigen, und ist es gut, Kohärenz anzustreben ? Indem wir mit Widersprüchen leben, diese transparent machen, werden sie im interaktiven dynamischen Prozess - vielleicht - kleiner.

Zögern, Missverständnisse aber auch Stereotypie fielen als Stichwörter beim Versuch, dem Wesen der Kohärenz näherzukommen. Gerade im Falle Indonesiens als Land im Aufbruch stellt sich die Frage sehr konkret und realitätsnah, wie die Instrumente der Entwicklungshilfe und der Wirtschaftsförderung abgestimmt werden müssen. Wollen wir eine bessere Kohärenz erreichen, müssen wir Antworten finden, die über die althergebrachten Schemen 
hinausgehen. Kohärenz in den Aussenbeziehungen bedeutet, dass sich alle auf ein Land gerichteten Beziehungen einem gemeinsamen Ziel unterordnen. Einzelne Handlungen dürfen nicht im krassen Widerspruch zum gemeinsamen Ziel stehen, auch dann nicht, wenn die Aktivitäten der verschiedenen Akteure untereinander nicht koordiniert sind. „Kohärenz bedeutet, dass erstens die linke Hand weiss, was die rechte tut - also Transparenz - und zweitens, dass mit der einen Aktion nicht die Arbeit der anderen zerstört wird“ (Violette Ruppanner). Kohärenzsuche ist ein Optimierungsprozess.

Die Konfliktforschung, die sich mit Regionalkonflikten in der südlichen Hemisphäre befasst, stellt fest, dass die Konfliktlinien zahlreicher und zum Teil auch tiefer werden. Es muss sorgfältig abgewogen werden, welche Auswirkungen eine für uns kohärente Politik beim Adressaten haben. Im sozio-kulturellen Bereich ist das Überwinden von Spaltungen und Konfliktlinien ein zentrales Element, um zu Demokratie, Wohlstand, Respekt der Menschenrechte, Schutz der Umwelt zu gelangen. „Eine Politik wäre also dann nicht kohärent, wenn sie Spaltungslinien zusätzlich erschwert, beispielsweise dadurch, dass sie einseitig einen bestimmten Sektor oder bestimmte Akteure bevorzugt und andere aus dem Blickfeld verliert" (Günther Bächler).

Wer das anzustrebende Ziel definiert - die Partner in Indonesien, die Akteure in der Schweiz, gemeinsame Zielformulierung - und welches dieses Ziel im Fall Indonesien sein könnte, wird in weiteren Konsultationen festzulegen sein. Einzelne - widersprüchliche - Ansätze sind vorhanden: „Es geht darum, das Ziel einer ganzheitlichen kohärenten Entwicklungspolitik anzustreben und insbesondere die wirtschafts- und handelspolitischen Instrumente stärker auf die entwicklungspolitischen Ziele auszurichten“ (Richard Gerster). Dieser Ansatz steht jedoch im Widerspruch zum liberalen Ansatz des "laisser faire“ der Privatwirtschaft.

\section{Welchen Prozess, welche Strukturen wählen wir?}

In einem ersten Schritt scheint ein grosser Handlungsbedarf in der Schweiz vorhanden zu sein. Es zeigt sich das Bedürfnis nach einem systematisierten Informations- und Konsultationsprozess in der Schweiz. Dieser schafft vermehrte Transparenz über die Zielkonflikte. Gerade in der Schweiz neigen wir dazu, alles immer harmonisch darzustellen. Oft wird die Entwicklungspolitik dazu benützt, um Innenpolitik zu treiben. Das verhindert die klare Analyse der Gegensätze in der Entwicklungspolitik, in der Aussenpolitik. „In diesem Sinne sehe ich Kohärenz als einen Prozess der Befreiung der Aussenpolitik von der Innenpolitik" (Matthias Meyer).

Haben wir die notwendigen Strukturen, um kohärente Politik zu machen ? Wirtschaftspolitik wird von einem Departement gemacht, Aussenpolitik und Entwicklungshilfe von einem anderen. Für einige Aussenstehende scheint die Koordination zwischen den beiden Departementen nicht immer gut zu funktionieren. Es drängt sich die Frage auf, welche Strukturbereinigung wir vornehmen sollten, um möglichst hohe Kohärenz zu erreichen. Dem wird entgegengehal- 
ten, dass die Schweiz mit ihrer systemimmanenten Konsenssuche geradezu ideale Strukturen hätte, um kohärente Politiken zu formulieren. „Es gibt kein westeuropäisches Land, das den Konsens so stark ins System eingebaut hat. Die Bedingungen für eine kohärente Politik wären ideal angesichts der internen Verwaltungsverfahren und der ausserordentlich ausdifferenzierten Konsultationsgesellschaft, die wir haben" (Peter Maurer). Warum ist dem nicht so ? Die Antwort wird im politischen Bereich gesehen. Kaum ein zweites Land hat wie die Schweiz seit jeher eine dermassen scharfe Trennung vorgenommen zwischen Politik und Wirtschaft. Wirtschaftlich und finanziell ist die Schweiz eine Grossmacht, politisch hat sich die Schweiz vom Diskurs in der Welt verabschiedet. Deshalb kommt es nicht zu den Entscheiden, die von den Strukturen her möglich wären.

„Kohärenz bedeutet, dass alle Akteure grosso modo am gleichen Strick ziehen, ohne dass dies unbedingt sehr koordiniert sein muss. Hauptsache wäre, dass es in die Richtung geht, wie sie erwünscht wird, denn wenn es dem Land wirtschaftlich dazu verhilft, auf der Entwicklungsstufe weiterzukommen, wird dies auch die Entwicklungsorganisationen und die Hilfswerke befriedigen, weil es dem Land insgesamt besser gehen kann, nicht muss, es gibt da natürlich noch die Verteilungsproblematik" (Max Schieler).

Die Diskussion am runden Tisch über die Beziehungen der Schweiz mit Indonesien konzentrierte sich stärker auf die Aktionsebene in der Schweiz und weniger auf den Handlungsbedarf, den wir von den Partnern in Indonesien erwarten. Klar formuliert wurde das Bedürfnis nach mehr Transparenz und für ein Instrumentarium, welches die Entscheidungen der einzelnen Akteure im Kohärenzprozess positiv beeinflusst.

\section{Durchsetzung der Kohärenz: mit welchen Mechanismen?}

Das Ziel, eine bessere Kohärenz der Politiken in der Entwicklungszusammenarbeit zu erreichen, erfordert wirksame Durchsetzungsmechanismen. Im Rahmen des Kolloquiums wurde diese Frage unter mehreren Gesichtspunkten behandelt:

a) Welches ist die Rolle dieser Mechanismen (Information, Konsultation, Mitentscheidung)?

b) Welches sind die von diesen Mechanismen betroffenen Akteure?

c) Genügen die bestehenden Mechanismen oder sind neue erforderlich?

\section{a) Die Rolle der Mechanismen}

Eine erste Feststellung fand rasch Einstimmigkeit: Ein guter Informationsaustausch ist eine wichtige Voraussetzung für das Streben nach grösserer Kohärenz der Politiken in der Entwicklungszusammenarbeit. Im Fall der schweizerischen Entwicklungszusammenarbeit mit Indonesien wurde bedauert, dass die 
Entscheidung des Ausstiegs der DEH und die Beauftragung des BAWI mit der Weiterführung der Zusammenarbeit nicht zur rechten Zeit mit den interessierten Kreisen (NGO, Privatwirtschaft) besprochen wurde.

Der Zugang der betroffenen Akteure zur Information über die Pläne der anderen Akteure gestattet es, die möglichen Widersprüche zwischen den Politiken und die Interessenkonflikte zwischen den verschiedenen Akteuren besser zu identifizieren. Die Konsultation ermöglicht es somit, Entscheide in besserer Kenntnis der Sachlage zu fällen und dabei gleichzeitig die Autonomie der Entscheidungen der einzelnen Akteure zu wahren.

Soll man über Informationsaustausch und Konsultation hinausgehen bis zu einer Kohärenzprüfung, die von allen Akteuren (Verwaltung, NGO, Privatwirtschaft) gemeinsam durchgeführt wird? Dies ist die These von Günther Bächler, welcher die Ansicht vertritt, dass Transparenz lediglich eine Vorbedingung ist für die Einsetzung von Kohärenzprüfungsmechanismen unter dem Gesichtspunkt der im Nord-Süd-Leitbild aufgeführten verschiedenen Ziele der Schweizer Politik. Diese Kohärenzprüfung betrifft nach Meinung Bächlers nicht nur die Bundesverwaltung, sondern auch alle anderen Akteure: „Die verschiedenen Akteure müssten im Grunde genommen diese Palette von Verträglichkeitsprüfungen in ihrem jeweiligen Bereich vornehmen und dann in den Dialog mit den übrigen Akteuren einbringen. Dann müsste man zu einer Optimierung dieser verschiedenen Strategien oder Pläne in Bezug auf die Kohärenz kommen".

Andere Teilnehmer sind der Ansicht, das man nicht über die Konsultation hinausgehen sollte. Nach Matthias Meyer „ist das eine, dass die interessierten Kreise sich gegenseitig über ihre Pläne informieren und dass dieser Austausch das Fällen von Entscheiden erleichtert. Das andere ist, durch Kohärenzprüfungen Einfluss auf die zuständigen Gremien nehmen zu wollen. Diese Kohärenzprüfung muss im wesentlichen in der Bundesverwaltung stattfinden. Der Konsultationsprozess mit privaten Kreisen muss besser organisiert werden“. Die Konsultation darf auch nicht die Verantwortung der Gremien verwässern, welche Entscheidungen zu treffen haben.

\section{b) Die Akteure}

In ihren schriflichen Beiträgen zum Kolloquium haben die Teilnehmerinnen und Teilnehmer je nach den betroffenen Akteuren unterschiedliche Mechanismen identifiziert oder vorgeschlagen. Man findet dort einerseits die internen Mechanismen der Bundesverwaltung und andererseits die Mechanismen, die andere Akteure in der Schweiz oder in Indonesien einbeziehen.

Was die Bundesverwaltung betrifft, so haben mehrere der Verwaltung nicht angehörende Diskussionsteilnehmer den Wunsch ausgesprochen, dass die Koordination zwischen den beiden hauptsächlich betroffenen Bundesämtern, DEH und BAWI, verstärkt werden solle. Ein Teilnehmer ging so weit, eine Zusammenlegung des Entwicklungsdienstes des BAWI mit demjenigen der DEH vorzuschlagen. Jedenfalls wird es als wünschenswert und möglich erachtet, die 
Aktivitäten der beiden Bundesämter im Rahmen der Koordinationsbüros der Schweizer Entwicklungszusammenarbeit in den Schwerpunktländern der schweizerischen Entwicklungshilfe stärker zu integrieren. In der Bundesverwaltung sollte die Koordination von Aussenpolitik und Aussenwirtschaftspolitik ohne grosse Schwierigkeit vorgenommen werden können, da in den meisten Entwicklungsländern die Aussenpolitik der Schweiz praktisch mit ihrer Politik der Entwicklungszusammenarbeit zusammenfällt.

Die Beteiligung der Privatwirtschaft an den Konsultationsmechanismen wirft eine Grundsatzfrage auf. Für Andreas Zürcher „spielt die Privatwirtschaft heute eine wichtige Rolle, doch ist ihre Rolle in der schweizerischen Entwicklungspolitik nicht definiert“. Solange sie nicht definiert ist, sehen die Unternehmen keinen Sinn, Information in Umlauf zu bringen. Da die Firmen in den Entwicklungsländern eine wichtige Rolle spielen, geht es darum, sie in den Prozess des Strebens nach Kohärenz mit einzubeziehen. Hierfür ist es notwendig, dass die anderen Akteure die Rolle anerkennen, welche die Privatwirtschaft in der Entwicklung spielen kann und dass man die alten Streitigkeiten und Anschuldigungen beiseite lässt. Der Dialog wird schwierig sein, wenn die Rolle der Privatwirtschaft ständig in Frage gestellt und kritisiert wird. Man muss sich auch dessen bewusst sein, dass die Überlegungen in den Unternehmen über die Rolle, die sie in der Entwicklungspolitik spielen können, noch nicht sehr weit fortgeschritten sind. Wenn diese Bedingungen jedoch erfüllt sind und ein Klima gegenseitigen Vertrauens besteht, dann wird ein richtiger Dialog stattfinden können, weil jeder Akteur gute Gründe hat, nach grösserer Kohärenz zu streben.

Philippe Régnier erscheint es besonders wichtig, die kleinen und mittleren Unternehmen der Schweiz an der Entwicklungszusammenarbeit mit Indonesien teilhaben zu lassen. Die schweizerischen Klein- und Mittelbetriebe sind auf den aussereuropäischen Märkten nicht sehr aktiv, und gemäss der OECD ist die Schweiz im Vergleich zu anderen Industrieländern im Rückstand, was die Förderung dieser Kategorie von Unternehmen betrifft. Die von NGO wie SWISSCONTACT und FUNDES unternommenen Anstrengungen zur Förderung der kleinen und mittleren Unternehmen in den Entwicklungsländern haben kein genügendes Echo gefunden. Die offiziellen Förderungsinstrumente sollten evaluiert werden. Beim BAWI werden derzeit Projekte geprüft, um Schulung und "Joint Ventures" sowie den Umwelttechnologietransfer zu fördern. Ferner ist eine Studie in Bearbeitung mit dem Ziel, eine schweizerische Investitionsgesellschaft für Entwicklung zu errichten, welche „Joint Ventures“ durch Darlehen, Kapitalbeteiligungen und Garantien fördern sollte. Die interessierten Kreise sollen darüber konsultiert werden.

Das Streben nach Kohärenz soll auch dadurch gefördert werden, dass man die Kontakte und den Dialog mit den Partnern in Indonesien intensiviert. Mit den traditionellen Partnern der Schweiz (öffentliche Institutionen und NGO) sollte eine besondere Anstrengung unternommen werden, um sich besser darüber zu informieren, was sie von den verschiedenen schweizerischen Akteuren erwarten. Desgleichen sollte der Suche nach neuen Partnern Priorität einge- 
räumt werden, und zwar nach Ansicht von Robert Jenny insbesondere mit jenen Partnern, die aus dem zur Zeit in Indonesien erfolgenden Denzentralisierungsprozess hervorgehen.

\section{c) Welche Mechanismen?}

Sind die bestehenden Mechanismen ausreichend oder muss man neue Mechanismen vorsehen? Matthias Meyer ist der Meinung, dass man das Bestehende besser ausnützen sollte, anstatt weitere Konsultationsgremien zu schaffen.

Der „Policy Dialogue“ mit den indonesischen Partnern ist ein Mechanismus, dessen Nützlichkeit nicht bestritten wird. Jedes Treffen kann Gelegenheit bieten, einen Dialog über diejenigen Themen zu führen, die den verschiedenen Akteuren der bilateralen Beziehungen wichtig erscheinen. Die NGO werden bereits bei der Überlegung der Bundesverwaltung über die im Rahmen der Bretton Woods-Institutionen verfolgte Politik herangezogen. Eine weitergehende Einbeziehung der NGO in den "Policy Dialogue" ist prinzipiell möglich, jedoch ist es vom Standpunkt der Bundesverwaltung nicht immer leicht, die NGO zu wählen, mit denen man einen Dialog führen sollte, da sie zahlreich sind und oft auseinandergehende Standpunkte haben.

Die Wirtschaftskammern sind ein sehr nützliches Konsultationsinstrument, da es schwierig ist, in einem ad hoc Rahmen mit einzelnen Unternehmen einen Dialog zu führen. Die Wirtschaftskammer für Südostasien wird aufgrund ihrer Organisation die für jedes Mitgliedsland spezifischen Fragen berücksichtigen können $\left({ }^{\star}\right)$. Die Beteiligung von Vertreterinnen und Vertretern akademischer Kreise und der Bundesverwaltung wird es der Wirtschaftskammer für Südostasien gestatten, als Forum für einen Dialog über Kohärenz zu dienen.

Sollten die Besuche von Bundesräten in einem Schwerpunktland der schweizerischen Entwicklungszusammenarbeit nicht "Höhepunkte" der Absprache mit allen schweizerischen Akteuren sein? Im Zusammenhang mit dem Besuch von Bundesrat Delamuraz in Indonesien im Oktober 1994 hätte der Schweizerische Gewerkschaftsbund, gemäss einer Erklärung von Margrit Meier, gerne einige Auskünfte über die Lage der Gewerkschaften und die Einhaltung der Menschenrechte in diesem Land übermittelt.

Unter den Neuerungsvorschlägen, die von einigen Teilnehmern vorgebracht wurden, wurden zwei Vorschläge besonders diskutiert.

Nach Ansicht von Richard Gerster sollte man die Einführung von „symetrischen, langfristigen und kohärenten Zusammenarbeitsverträgen vorsehen, die einen allgemeinen Rahmen für alle Dimensionen der bilateralen Beziehungen festlegen. Derzeit schliesst die Schweiz mit Entwicklungsländern Abkommen über die verschiedensten Bereiche ab (Doppelbesteuerung, Investitionsschutz, Luftverkehr, Entwicklungszusammenarbeit). Die Aushand-

* Die Gründung der Wirtschaftskammer Schweiz-Südostasien erfolgte am 16. November 1994. 
lung eines langfristigen Rahmenabkommens der Zusammenarbeit böte die Gelegenheit, mit den Partnern des Schwerpunktlandes nicht nur alle Aspekte der Politik der Zusammenarbeit, sondern die Gesamtheit der bilateralen Fragen zu diskutieren, wobei man zum Beispiel gegebenenfalls die Migrationsprobleme mit einbezieht".

Dieser Vorschlag rief kritische Bemerkungen hervor in Bezug auf die Schwierigkeit, einen solchen Mechanismus einzusetzen, und über die mögliche Schwerfälligkeit eines derartigen Instruments. Einige Teilnehmer zweifeln daran, dass die Schweiz im Fall Indonesiens genügend Gewicht habe, um auf das Partnerland auch nur irgendwelchen Einfluss auszuüben. Die kritische Masse ist bei weitem nicht erreicht. Die Idee, mit einem Partnerland eine Verhandlung durchzuführen, wie sie die Europäische Union mit den Partnerstaaten der Lomé-Abkommen führt, scheint seitens der Schweiz schwer realisierbar, ausser eventuell mit einem kleinen Partnerland, mit dem die bilateralen Beziehungen besonders bedeutend sind. Man stellte fest, dass der multilaterale Rahmen es auch der Schweiz gestattet, sich bei den Partnerländern Gehör zu verschaffen. Jedoch wurde zugegeben, dass die Schweiz keinen Einfluss auf die Politik Indonesiens über bilaterale Konditionalitäten ausüben kann.

Der Vorschlag, eine dreigliedrige nationale Kommission (bestehend aus Bundesverwaltung, Privatwirtschaft und Universitäten) als Mechanismus zur Koordination und Zusammenarbeit zu schaffen, wurde mit Interesse aufgenommen, wobei festgestellt wurde, dass eine solche Kommission auch die NGO mit einschliessen könnte. Jedoch wurden einige grundsätzliche Einwände formuliert. Man müsste über ähnliche Kommissionen für alle wichtigen Länder verfügen; könnte diese Vielzahl von Kommissionen von den betroffenen Institutionen verwaltet werden? Hans-Peter Maag fragte sich, ob solche Kommissionen es nicht unmöglich machen würden, die Zusammenarbeit mit dem betreffenden Land zu reduzieren oder einzustellen, während man anderweitig von der DEH fordert, ihre Entwicklungszusammenarbeit stärker zu konzentrieren.

\section{Schlussfolgerungen}

Diese Kohärenzdiskussion, die auf dem Beispiel der schweizerischen Entwicklungszusammenarbeit mit einem grossen und bedeutenden Land beruhte, muss als erster Ansatz einer komplexen Problematik betrachtet werden. Sicherlich müssen die Überlegungen konzeptuell wie auch bezüglich der Durchsetzungsmechanismen noch vertieft werden. Jedoch scheint es den Verantwortlichen dieses Dossiers, dass zu einigen wichtigen Punkten Schlussfolgerungen gezogen werden können, die den Weg weisen, um die praktische Umsetzung des im Bericht des Bundesrates befürworteten Ansatzes voranzutreiben.

- Der Austausch der Informationen über Anlaysen und Projekte unter den von der wirtschaftlichen und sozialen Entwicklung eines Partnerlandes der 
Schweiz betroffenen Akteure in einem ad hoc Forum ist nicht nur für das Streben nach mehr Kohärenz in den Politiken notwendig, sondern auch, um die Qualität der Entscheidungen der verschiedenen Akteure zu verbessern. Wenn die Meinungen bezüglich der Möglichkeit bzw. der Zweckmässigkeit, bei der Kohärenzprüfung der Aktionen der verschiedenen Akteure weiter zu gehen, auch auseinandergehen, so wird doch anerkannt, dass ein solcher Austausch notwendig ist, und alle Diskussionsteilnehmer haben ihre Bereitschaft bekundet, daran teilzunehmen.

- Eine Voraussetzung dafür, dass ein solcher fruchtbarer Dialog stattfindet, ist die klare Definition der Rolle eines jeden Akteurs in der Entwicklungszusammenarbeit. Dies ist heute noch nicht der Fall, insbesondere was die Rolle der Privatwirtschaft betrifft. Es ist daher wichtig und dringlich, diese Lücke zu schliessen, damit der Dialog über Kohärenz vorankommt.

- Eine weitere Bedingung für die Verstärkung der Überlegungen über Kohärenz ist in der Schweiz der Ausbau der Forschung und Ausbildung in der Asienkunde. Hierdurch könnten die künftigen Akteure gleich vom Anfang ihrer Laufbahn an über die Entwicklungsprobleme Asiens informiert werden und der Problematik der Kohärenz aufmerksam gegenüberstehen. Unter Berücksichtigung der beschränkten menschlichen und finanziellen Ressourcen könnten die akademischen Kreise im Einverständnis mit den anderen Akteuren eine „Beobachtungsstelle“ errichten, welche die von den spezialisierten Institutionen in aller Welt hervorgebrachten Forschungsdaten und ergebnisse zusammentragen und für die schweizerischen Akteure sachdienlich verwendbar machen würde.

Die wichtigste Schlussfolgerung des Kolloquiums ist zweifelsohne, dass alle schweizerischen Akteure der Beziehungen zu Indonesien gute Gründe haben, dass sich die Kohärenz der Politiken verbessert. Sie müssen jetzt das Forum finden, um die Überlegungen fortzusetzen und die indonesischen Partner in einem möglichst weitgesteckten Rahmen daran teilhaben zu lassen.

Das Jahrbuch Schweiz-Dritte Welt wird seinerseits Beiträgen zu diesem Thema immer offenstehen. Diese erste Prüfung der konkreten Probleme, die sich bei der Durchsetzung kohärenterer Politiken ergeben können, hat in der Tat gezeigt, dass die Akteure, die wir zum Kolloquium eingeladen haben, die Nützlichkeit dieses Vorgehens anerkannt und mit Aufgeschlossenheit daran teilgenommen haben, indem sie Klischees und vorgefasste Meinungen beiseite liessen. 